\title{
Growth mechanism of Herring-Bone and Hour-glass synthetic gypsum
}

\author{
D JAYAKUMAR and K S RAJU* \\ Department of Applied Geology, University of Madras, Guindy Campus, \\ Madras 600025 , India \\ *Department of Crystallography and Biophysics
}

MS received 20 December 1982; revised 5 February 1983

\begin{abstract}
Synthetic gypsum crystals are grown from sodium meta silicate gel when $1 \mathrm{M}$ calcium chloride solution diffuses into the gel imbedded with $1 \mathrm{M}$ amonium sulphate solution. Acicular-shaped crystals of Herring-Bone structure are observed. When methanol was added to the gel medium, tabular crystals revealing Hour-glass structure are rarely abserved. In the presence of methanol the silicate particles are inhibited from being incorporated into the crystals as a result of pushing and incorporating mechanisms. Inclusion of silicate particles in traces gives rise to Hour-glass pattern. The implications are discussed.
\end{abstract}

Keywords. Gel grown gypsum;Herring-Bone structure;Hour-glass structure; synthetic gypsum.

\section{Introduction}

Single crystals of very high solubilities can be grown by slow evaporation of super saturated solutions. But those with low solubilities can hardly be grown from solution, as the supply of the dissolved component is limited. Gel medium is used to grow the crystals sparingly soluble in water derived from the diffusion of reactants of very high solubilities. Large crystals can be grown in gel as supersaturation is much higher in the gel than in the solution. This has been attributed to the nucleation suppressing character of the gel according to Henisch (1970). Henisch et al (1965) reported that calcium tartrate tetrahydrate crystals, only sparingly soluble in water, are grown by imbedding tartaric acid in the gel and allowing $1 \mathrm{M}$ solution of calcium chloride to diffuse into the gel. The surface topography and habit of gypsum grown in two different gel media i.e., sodium silicate gel and bentonite clay gel have been reported by Cady and Shanks (1974). Van Rosmalen et al (1976) studied the morphology of gypsum grown in silica and agar gels in the presence of additives. Lattice perfection has been studied from the $\mathrm{x}$-ray Laue transmission pattern, which always consists of very sharp spots, while the pattern of opaque equants consisted of elongated spots in a radial direction. Displacement of sand particles in sandy gypsum crystals has been studied by Shearman (1981). Displacement of sand grains 
by mechanical force overcoming the resitance of inclusion has given rise to HerringBone structure. Kastner (1970) has reported Hour-glass pattern in the synthetic gypsum by the incorporation of calcite inclusion.

The present work deals with studies on the growih of gypsum in sodium meta silicate gel and alcoholic gel.

\section{Experimental}

The work is in two stages. First, sodium meta silicate solution (Sp.Gr. $1.06 \mathrm{~g} \mathrm{~cm}^{-3}$ ) is prepared and its $\mathrm{pH}$ adjusted to 7.5 by adding $50 \%$ acetic acid. Twenty $\mathrm{ml}$ of this is poured in a test tube ( $40 \mathrm{~mm}$ diameter) to which is added $20 \mathrm{ml}$ of $1 \mathrm{M}$ ammonium sulphate solution. The gel is allowed to set and $20 \mathrm{ml}$ of $1 \mathrm{M}$ calcium chloride solution are added in drips through the walls of the test tube so that the gel is undisturbed. The crystals are harvested after two weeks. Secondly, methanol is added to the gel in quantities of 2 to $20 \mathrm{ml}$ in steps of $2 \mathrm{ml}$ before adding ammonium sulphate solution (20 ml) and the whole experiment is repeated as in stage I described above. The test tubes are kept undisturbed. The tiny crystals appeared within a month and these were confirmed as gypsum by using the following methods.

\subsection{Chemical analysis}

Calcium sulphate dihydrate (gypsum) single crystals are derived by the diffusion of calcium chloride into the gel containing ammonium sulphate solution, according to the chemical reaction

$$
\mathrm{CaCl}_{2}+\left(\mathrm{NH}_{4}\right)_{2} \mathrm{SO}_{4} \rightarrow 2 \mathrm{NH}_{4} \mathrm{Cl}+\mathrm{CaSO}_{4}
$$

The crystals harvested from the gel are given an acetone bath. These crystals on water treatement are highly insoluble, which agrees with the low solubility of $\mathrm{CaSO}_{4}-2 \mathrm{H}_{2} \mathrm{O}$ in water $(0.241 \mathrm{~g}$ being soluble in $100 \mathrm{ml}$ of water $)$ reported earlier (Anon 1977-78).

\subsection{IR absorption studies}

The IR spectrum of the crystalline sample revealed strong absorption peaks relating to sulphate group $\left(1200 \mathrm{~cm}^{-1}\right)$ and to lattice water $\left(3000-3700 \mathrm{~cm}^{-1}\right)$, suggesting that the crystalline samples are gypsum.

\subsection{Cleavage and etching}

The well-defined $(010)$ basal cleavage is revealed by the crystals reported here and on etching to cleavage with analar grade nitric acid, rhombohedral pits are formed which agrees with the findings of Raju (1981) on natural gypsum. 


\subsection{VHN and Laue $x$-ray diffraction pattern}

On indenting the $(010)$ cleavage of synthetic gypsum with $1 \mathrm{~g}$ load, the VHN has been found to be $17.96 \mathrm{~kg}$ per $\mathrm{mm}^{2}$ and the Laue $\mathrm{x}$-ray transmission diffraction pattern on the synthetic gypsum reported here agrees very well with the earlier findings of Patel and Raju (1970) on natural gypsum.

\section{Observation and discussion}

From the crop of the crystals obtained from the first stage of the experiment (without imbedding alcohol in the gel), needle-shaped crystals emerged in the gel from interface. Figure 1 shows crystals having dimension $12 \times 2 \times 2 \mathrm{~mm}$. The crystals are hazzy, whitish, needle-shaped and rough surfaced. The crystals are translucent and whitish in colour which suggest that the gel might have got included in the crystal. This has been confirmed by dissolving the crystal in concentrated nitric acid, wherein large particles of gel are clearly observed. The Laue transmission $x$-ray diffraction pattern of the crystal reveals a Herring-Bone structure (figure 2) indicating a smaller number of spots with more extended streaks and further confirms that gel inclusion assists in the formation of the Herring-Bone structure.

During the crystal growth, two mechanical forces come into picture (i) The force which tries to keep the gel particles away from getting imbedded and (ii) the force which accommodates the gel particles. In the Herring-Bone structure, the latter force is dominant, and hence the amount of gel imbedded is relatively larger. This agrees with the findings of Shearman (1981).

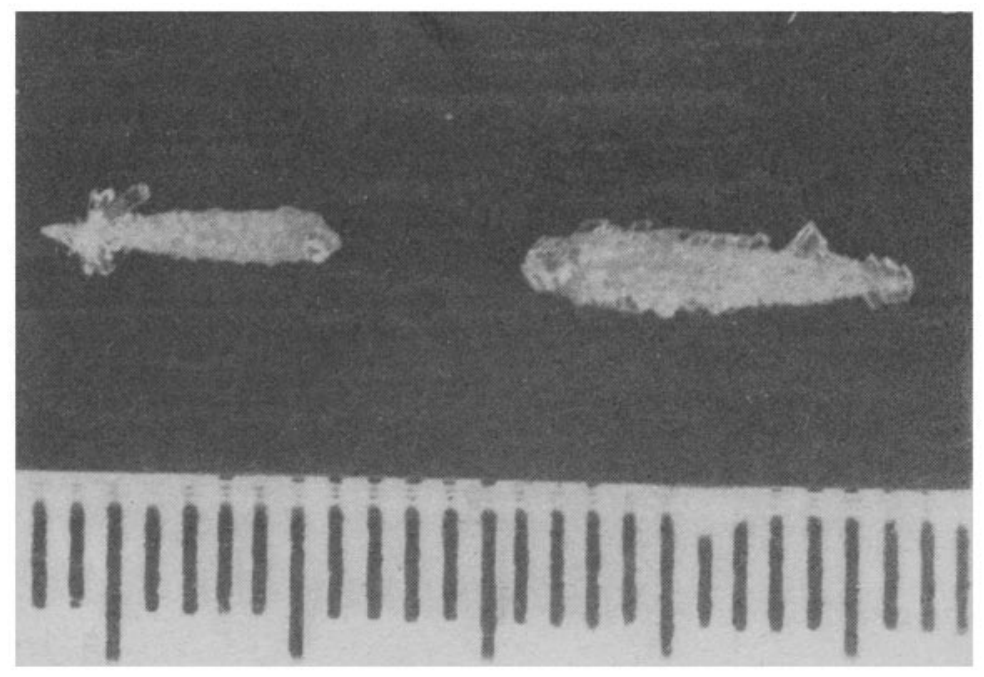

Figure 1. Gypsum crystals revealing Herring-Bone structure ( $\mathrm{mm}$ scale). 


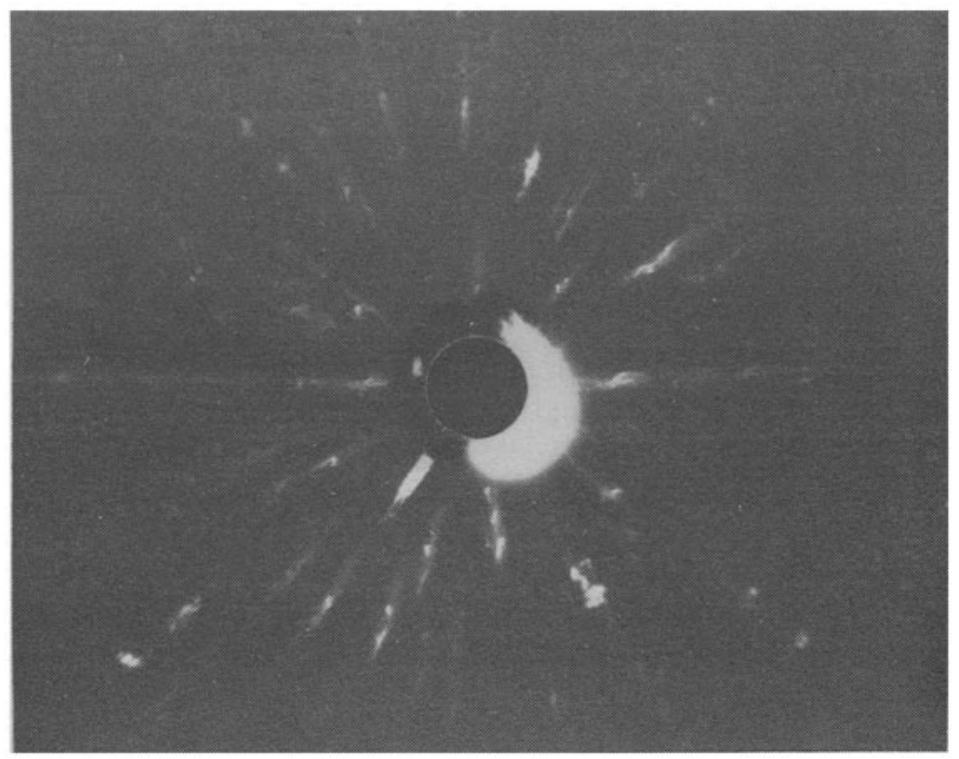

Figure 2. Laue $x$-rap transmbsion diffraction of the Herring-Bone structure.

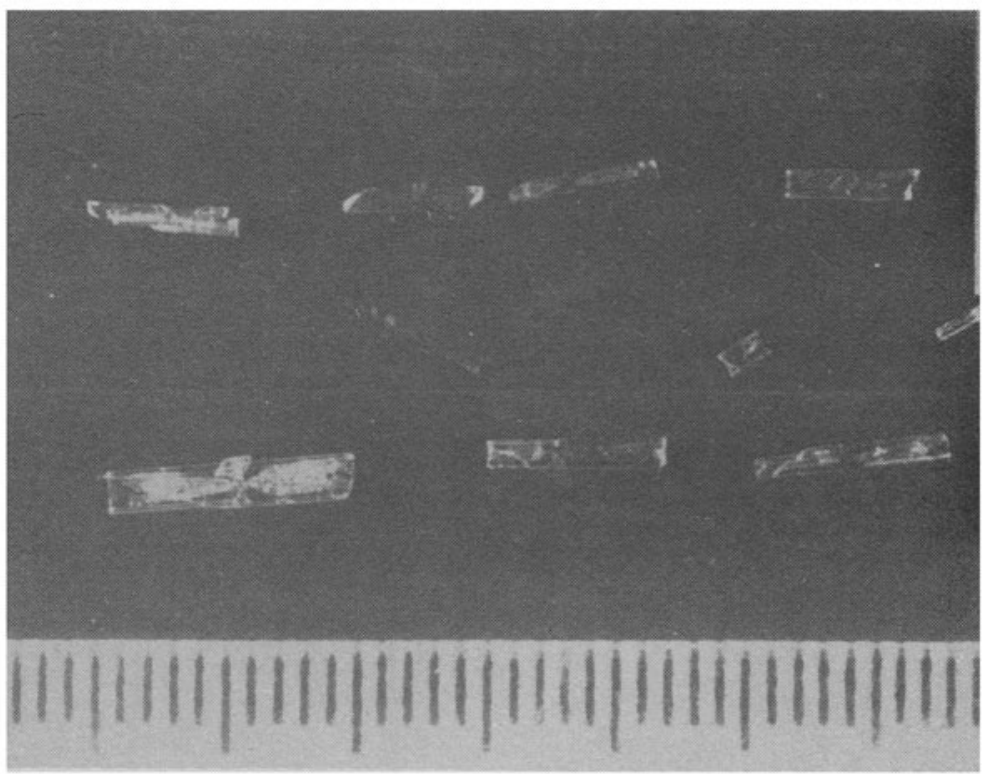

Figure 3. Gypsin arstal revaline Hour-glass tructure 


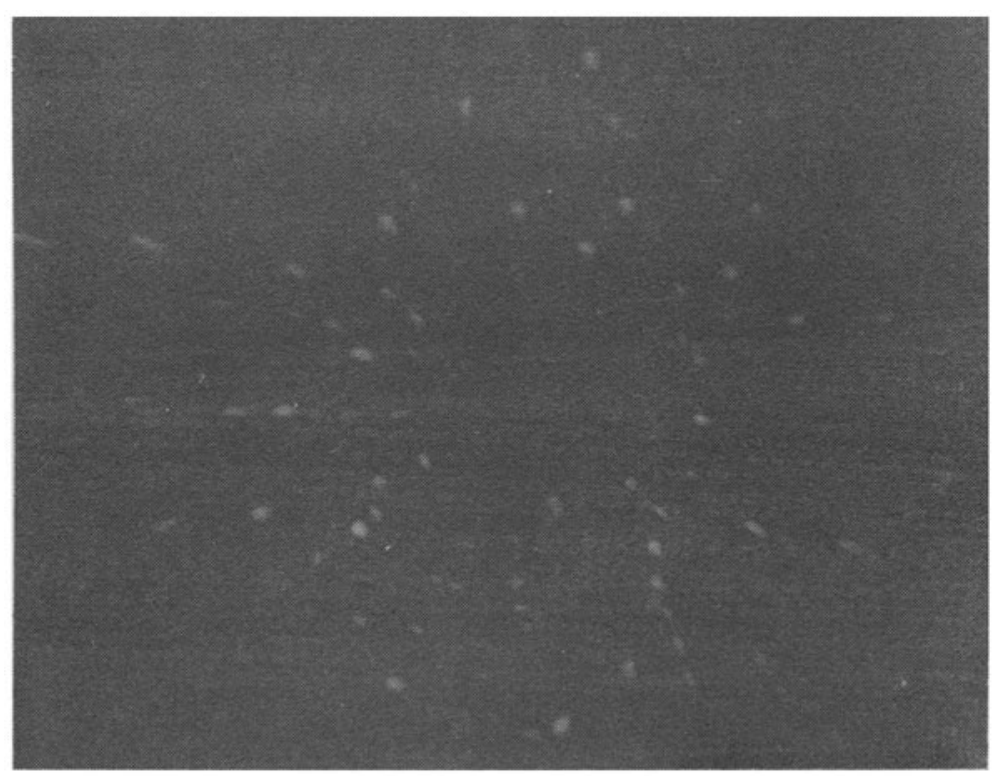

Figure 4. Laue $x$-ray transmission diffraction of the Hour-glass structure.

Figure 3 is the photomicrograph of an 'Hour-glass' structure of size $9 \times 2 \times$ $1 \mathrm{~mm}$, when the gypsum crystal is grown in gel having $10 \mathrm{ml}$ of methanol. This crystal is grown at a greater depth in the gel and is more transparent and less hazzy. Therefore, the gel included in the crystal is limited to a greater extent due to the relative aging of the gel at greater depths as well as the presence of methanol. When the crystal, which reveals the Hour-glass structure. is dissolved in concentrated nitric acid, very minute traces of gel are observed. The Laue $x$-ray transmission diffraction pattern taken over tabular crystals revealing Hour-glass pattern (figure 4) consisted of more spots with less extended streaks than the crystal having the Herring-Bone structure described above.

Synthetic gypsum from calcite powder and sea-water shows inclusion giving rise to Hour-glass patttern. The pushing and incorporating mechanism of calcite particles as a function of growth rate may be responsible for the formation of an inclusion Hour-glass pattern, according to Kastner (1970).

In the present case, gel particle play the role of calcite particles in the growth of natural gypsum. The silicate particles of the gel get included in the synthetic gypsum in a limited way, due to methanol, controlling both the nucleation centres and gel inclusion and as a result of pushing and incorporating mechanisms gives rise to Hour-glass structure, resembling the pattern reported by Kastner (1970).

\section{Conclusion}

The gel grown gypsum crystals, without imbedding alcohol, gives rise to HerringBone structure, owing to random inclusion of gel (sodium meta silicate) particles, 
as established by hazzy whitish crystals as well as by the smaller number of more extt.dded streaks. On alcoholing the gel medium, gel inclusion in the crystal is limited as established by the transparency and also by the greater number of spots with less extended streaks giving rise to Hour-glass structure. These experimental results agree with the finding of Shearman (1981) and Kastner (1970).

\section{Acknowledgement}

The authors thank Prof. R Srinivasan and Prof. N Leelananda Rao for their encouragement.

\section{References}

Anon 1977-78 Handbook of Chemistry and Physics (ed.) Robert C Weast (Ohio; CRC Press Inc), 58th edn., p. B-101

Cady R D and Shanks H R 1972, J. Cryst. Growth 23275

Henisch H K 1970 Crystal Growth in Gels (Pennsylvania: University Press) Chap. 4, p. 73

Henisch H K, Dennis J and Hanoka J I 1965, J. Phys. Chem. Solids 26493

Kastner M 1970 Am. Miner 552128

Patel A R and Raju K S 1970 J. Phys. Chem. Solids 31331

Raju K S 1981 J. Mater. Sci. 162512

Shearman D J 1981 Geol. Mag. 118303

Van Rosmalen G M, Marchee W G J and Bennema P 1976 J. Cryst. Growth 35169 